\title{
SIGILLATA AFRICANA CON TEMA BÍBLICO ENCONTRADA EN LA PROVINCIA DE SALAMANCA
}

\author{
POR \\ MIGUEL GARCÍA DE FIGUEROLA y JOSÉ GARCÍA MARTÍN \\ Departamento de Prehistoria, Historia Antigua y Arqueologia. Universidad de Salamanca
}

\section{RESUMEN}

Se presentan aqui varios fragmentos de un único recipiente de sigillata africana con decoración aplicada a molde, encontrados en un yacimiento situado en el término municipal de Huerta (Salamanca). La temática decorativa es de carácter religioso, correspondiendo a dos episodios biblicos concretos: El sacrificio de Isaac y los tres hebreos salvados del horno.

\section{SUMMARY}

We present here several fragments from a single pot of African red slip ware with relief decoration. They were found at a settlement in the territory limits of Huerta (Salamanca). The decorative motives are of a religous character, corresponding with two specific biblical episodes: "Abraham sacrificing lsaac" and "The three hebrews saved from the fournace».

En 1993, en el transcurso de las prospecciones de yacimientos romanos llevadas a cabo por miembros del área de Arqueología, del departamento de Prehistoria/Historia Antigua y Arqueología de la Universidad de Salamanca, con vistas a la realización de un proyecto de investigación sobre el poblamiento rural romano ', se encontró un fragmento de sigillata africana, con decoración aplicada en relieve que representaba una escena con tema bíblico.

Pesquisas posteriores entre las colecciones lo-

' El citado proyecto, financiado por la DGICYT, lleva por título Implantación romana en el territorio de Salmantica (código PB 91-0419) y está dirigido por el Dr. Enrique Arif̂o Gil. Queremos agradecer aquí la ayuda prestada para la realización de este trabajo al Dr. Pablo Díaz Martínez y a la Dra. M. Teresa Amaré Tafalla. Nuestro agradecimiento también a D. Luis Angoso por su colaboración. cales, nos permitieron recuperar otros cuatro fragmentos de similares características, procedentes del mismo yacimiento. Hemos conseguido unir algunos entre sí encontrándonos con tres escenas decorativas colocadas en el borde de un gran plato, sin que exista la menor duda de que los cinco fragmentos pertenecen al mismo recipiente, como atestiguan, en último término, las lineas de torno visibles en su parte posterior. El presente articulo tiene por finalidad dar a conocer el hallazgo, cuyo principal interés reside en su excepcionalidad en el marco geográfico de la meseta norte ${ }^{2}$.

\section{EL YACIMIENTO}

Los fragmento ${ }^{3}$ proceden del yacimiento de Los Bebederos, situado a unos $12 \mathrm{kms}$. al este de Sala-

\footnotetext{
${ }^{2}$ Aunque la investigación hispana presenta un gran vacio en lo que se refiere a la publicación de hallazgos de sigillata africana $C$ en la meseta norte, nos atrevemos a pensar en que, aunque sin llegar a ser abundante, su comercio fue más intenso de lo que en principio pudiera creerse. A los hallazgos de Huerta podemos añadir los publicados de Avila (Niharra) y de Complutum y Valeria: Járrega Domínguez, R.: «Sigillata africana en la provincia de Avila: Hallazgos de Niharra" $A E A R Q 63,1990$, pp. 344-346. En este contexto, adquieren sentido los fragmentos de molde con temática cristiana encontrados en Zamora: López Rodriguez, J. R. y Regueras Grande, F.: "Cerámicas importadas tardorromanas de Villanueva de Azoague (Zamora)" BSAA, 53 , Valladolid, 1987, pp. 115-166.

${ }^{3}$ Dos de los cinco fragmentos - núms.1 y 4 - habian sido publicados con anterioridad por Pradales Ciprés, D.: «Nuevos hallazgos cerámicos de Terra Sigillata Hispánica en la provincia de Salamanca" Actas del Primer Congreso de Historia de Salamanca, t. I, Salamanca, 1992, p. 323, quien se limitó a presentarlos como fragmentos escepcionales de Terra Sigillata. Estos dos fragmentos, como el resto de las piezas aquí publicadas han sido depositadas en el Museo de Arqueología de Salamanca.
} 
manca, junto al municipio de Huerta, en las coordenadas geográficas I $46^{\circ} 58^{\prime \prime} / 4048^{\prime} 22^{\prime \prime}$.

Los Bebederos ocupan una superficie estimada de 3 has. de terreno llano, en la margen derecha del rio Tormes. El yacimiento, que nunca ha sido excavado, es considerado como uno de los varios asentamientos romanos de gran relieve situados en las fértiles tierras aluviales que se encuentran a ese lado de la ciudad. No se conocen estructuras y, al contrario de lo que ocurre con los yacimientos cercanos (San Lázaro en Aldealengua, La Aceña de la Fuente o Castañeda de Tormes ${ }^{4}$, nunca se ha constatado la presencia de mosaicos ni ningún otro resto monumental que nos haga pensar en la existencia de una villa.

La cronología de Los Bebederos, a juzgar por los hallazgos recogidos en superficie, es muy amplia, abarcando al menos desde el siglo ia.C. - como parecen atestiguar los hallazgos de cerámica de tradición indigena con decoración bicroma y los fragmentos de terra sigillata itálica-, hasta los siglos VI y VII, momento en que vienen datándose los abundantes fragmentos de pizarras grabadas, de tipo cursivo, numeral o con dibujos que aparecen en el yacimiento 5 .

La importancia de Los Bebederos como asentamiento de la época romana y altomedieval puede haber estado motivada, no sólo por su situación en relación con la calidad de las tierras, sino por la existencia de un vado sobre el Tormes en esas épo-

\footnotetext{
+ Estos yacimientos son bien conocidos por la bibliografia salmantina. Maluquer, J.: "Carta Arqueológica de Salamancan, Salamanca, 1958, pp. 108-109 (Aceña de la Fuente). También Garcia de Figuerola, M.: Prospección de villas romanas en Salamanca. Informe inédito. Biblioteca Museo de Salamanca, r.4328. Sobre la Aceǹa de la Fuente puede verse también el trabajo de Angoso. L. aEl asentamiento rural romano de El Cenizaln Revista Prov de Estudios 16-17. Salamanca, 1985, pp. 341 y ss. Sobre La Piñuela puede verse Arias, L. y Jimenez, M.: "Dos nuevos yacimientos romanos imperiales en la provincia de Salamanca” Rev. Prov. de Estudios, 8. Salamanca, 1983, p. 81 y ss.

'Algunas de estas pizarras han sido publicadas. Garcia Martín, J.: "Seis dibujos visigodos con instrumentos agricolas y animales domésticos sobre pizarras salmantinas" Revista. Prov. de Estudios 4, 1982, pp. 56-57. Santonja, M. y Moreno, M.: «Tres pizarras con dibujos de época visigoda en la provincia de Salamancan Zephirus 44-45, Salamanca, 1991-192, p. 471 y ss. También Velázquez, I. en su estudio sobre este tipo de soportes epigráficos: «Las pizarras visigodas: Edición critica y estudio». Univ. de Murcia/Univ. de Alcalá de Henares/Junta de Castilla y León, 1989, estudia en pp. 46 y 809 algunos ejemplares de Huerta.

Sobre algunos hallazgos numismáticos del yacimiento puede verse el articulo de de Figuerola,M. «Hallazgos numismáticos en la provincia de Salamanca (II y III)". Revista Prov. de Estudios, 26, Salamanca, 1990, pp. 237-254 (monedas del siglo III y IV). También el libro del mismo autor: Numismática Antigua de Salamanca (en preparación), donde se recogen otros hallazgos del siglo 1.
}

cas, que permitiria enlazar ambas orillas del rio evitando el rodeo que significaba utilizar el puente junto a Salmantica.

\section{DESCRIPCIÓN DE LOS FRAGMENTOS CERÁMICOS}

Fragmentos 1 y 2: Fragmentos de borde de un gran plato circular. Labio curvo. Presenta dos acanaladuras, situada la más externa a $0,4 \mathrm{~cm}$ del extremo del labio. Mide $6 \mathrm{~cm}$ de longitud mayor $\times 5,7 \mathrm{~cm}$ de ancho mayor. El grosor es de $0,6 \mathrm{~cm}$.

La decoración, en relieve aplicado a molde, consiste en una figura masculina de $5,5 \mathrm{~cm}$ de altura, vestida con túnica corta, que lleva una espada apoyada sobre el hombro izquierdo. El brazo derecho está semiestendido, con la mano abierta. No lleva calzado. La parte de la cabeza se ha desprendido, apreciándose únicamente su contorno.

Fragmento 3: Fragmento de borde de plato. No conserva el labio. Mide $5 \mathrm{~cm}$ de longitud máxima $\times 4,2 \mathrm{~cm}$ de ancho mayor. El grosor es de $0,6 \mathrm{~cm}$.

La decoración, en relieve aplicado a molde, muestra a la izquierda una figura masculina de pie, de complexión gruesa, barbada, mirando hacia atrás. Presenta un deconchado en el rostro y su altura es de $5 \mathrm{~cm}$. Viste túnica corta cogida con cinturón. Posiblemente lleve calzado, aunque no puede asegurarse pues falta la parte inferior de ambas piernas. Porta espada corta que mantiene a la altura del pecho. Con la mano izquierda sujeta la cabeza de un segundo individuo.

De éste se observa únicamente la cabeza y los hombros. Imberbe, de aspecto juvenil, vestido, yace sobre un altar con el rostro de perfil. Del altar se conserva la esquina superior izquierda, dando la impresión de ser cuadrangular, de corte pagano. Sobre el ara está representada la leña para el sacrificio.

Fragmentos 4 y 5: Fragmentos de borde de plato circular de grandes dimensiones. Labio curvo con dos acanaladuras situada la más externa a $0,4 \mathrm{~cm}$ del extremo del labio. Mide $9,4 \mathrm{~cm}$ de longitud máxi$\mathrm{ma} \times 6 \mathrm{~cm}$ de ancho mayor. El grosor es de $0,6 \mathrm{~cm}$.

La decoración presenta una escena en la que puede verse, a la izquierda, parte de una estructura arquitectónica. Está representado el aparejo isodomo ( 12 hiladas) y una puerta rematada en arco; no está enmarcada aunque si presenta umbral.

El ancho de la puerta está ocupado por una figura con forma de ángel o putti que sale hacia la derecha. A su alrededor se alzan 9 o quizás 10 «lenguas de fuego" que ascienden por los muros de la estructura arquitectónica. 


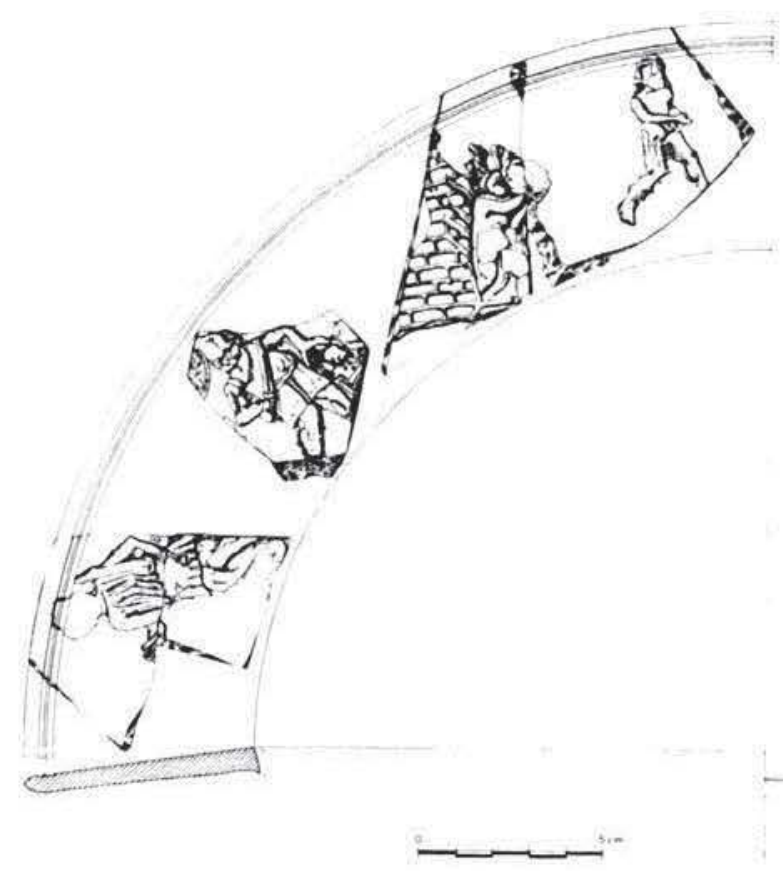

Figura I Fragmentos de Sigillatat africana de lluerta

La altura de la figura es de $4 \mathrm{~cm}$. Mantiene en el interior parte de la pierna izquierda. Lleva los carrillos hinchados en actitud de soplar. Delante de su rostro se ha dispuesto una masa más o menos circular en relación quizás con sus manos levantadas. La figura presenta desconchados en la cadera y la pierna izquierda.

A la derecha, puede verse otra figura masculina, barbada, avanzando a la derecha. Va desnuda y Ileva las manos por delante del cuerpo en actitud de súplica. Presenta rozaduras en el rostro y el hombro derecho. Le falta el pie izquierdo. Su altura es de $4,3 \mathrm{~cm}$.

\section{EL. RECIPIENTE CERÁMICO}

Los cinco fragmentos pertenecen genéricamente al tipo de cerámica denominada clara $C$, con decoración aplicada a molde.

Todos ellos corresponden a la misma forma cerámica - un plato de grandes dimensiones y al menos en tres de los fragmentos puede verse la misma acanaladura y el mismo labio curvo.

Técnicamente la pasta presenta el mismo grado de decantación y el barniz oscuro y brillante que permiten considerar que estamos ante una producción C3. Por otro lado, el estilo decorativo nos lleva
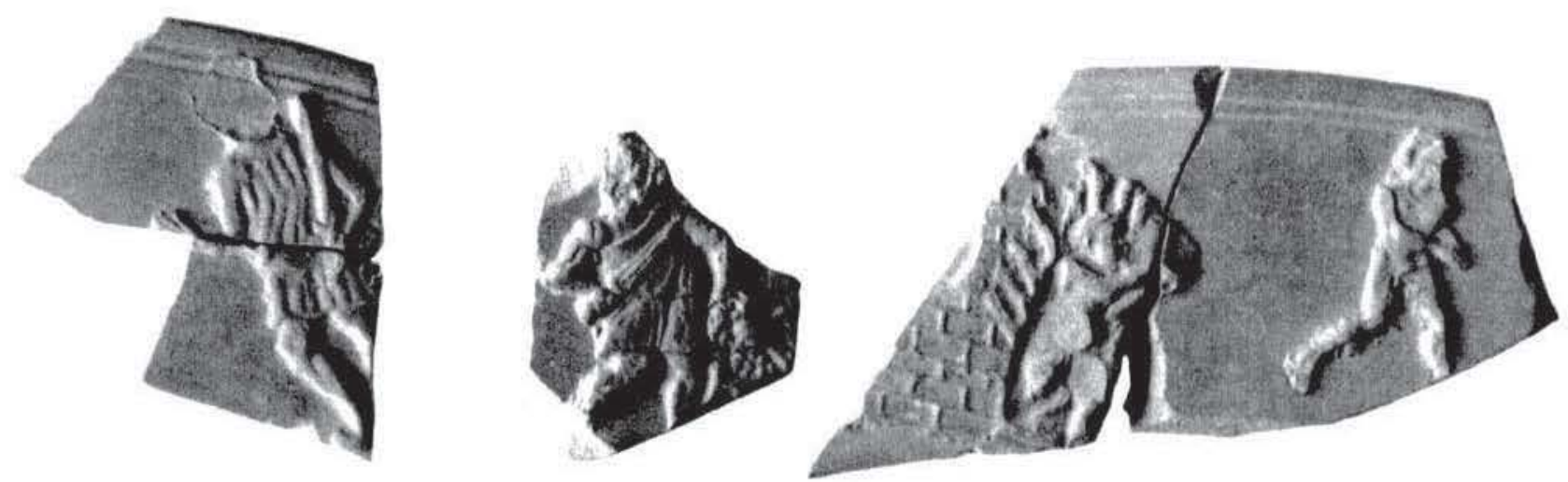

Figura 2.-De izquierda a derecha: Fragmentos 1 y 2 . Fragmento 3 y Fragmentos 4 y 5 . 
hasta el "estilo tardio" de Hayes ${ }^{n}$, situado por este investigador a partir de la segunda mitad del siglo IV y caracterizado por los motivos figurativos de gran tamaño y relieve, dispuestos en conexión con la forma del soporte.

Como hemos indicado con anterioridad, tal como atestiguan en último término las lineas de torno, no cabe duda de que los cinco fragmentos pertenecen al mismo recipiente: Un plato circular de grandes dimensiones $-40 \mathrm{~cm}$. de diámetro-, con el labio curvo, que suele asociarse a la forma 55 de Hayes y E de Salomonson ${ }^{7}$. La forma está representada en el Atlante (lam. LXXVI,3) y se documenta en Argelia, Italia y Tunicia ${ }^{*}$.

Hayes lo considera raro. Por lo que respecta a la cronologia, Salomonson lo situa a mediados del siglo Iv, mientras que para Hayes se fabrica entre la segunda mitad del siglo iv y la primera del v.

\section{INTERPRETACIÓN TEMÁTICA DE LA DECORACIÓN}

El primer tema decorativo (fragmentos 1 y 2 ) es generalizadamente reconocido como la escena preparatoria del sacrificio de Issac tal como aparece descrita en Génesis 22,6. El paralelo más conocido -y el más cercano- está en el plato procedente de Belo que se encuentra expuesto en el M.A.N. ${ }^{9}$ y que muestra en su interior dos figuras (Abraham e Isaac) junto con un cantharus y un árbol.

La figura de los fragmentos 1 y 2 del plato de Huerta es idéntica a la situada a la derecha en el recipiente de Belo, procediendo del mismo molde, como atestiguan en último término los pliegues de la túnica y el pomo de la espada. De esta manera

\footnotetext{
' Hayes, J. W., "Late Roman Pottery", Londres 1972, p. 214

7 Hayes, op. cit. 1972 p. 83 . Salomonson, J. W.: "Spätrömische rote tonware mit reliefverzierung aus nordafrikanischen werkstätten» BABesch., XLIV, 1969 presenta en figs. 22 y 32, dos fragmentos - de Hipona- de la forma $\mathrm{E}$.

"Vv.aa: "Atlante delle forme ceramiche I. Ceramica fine romana nell bacino mediterraneo (medio e tardo imperio). Enciclopedia dell arte antica classica e orientale» Roma, 1981. p. 158

- Este plato ha sido objeto de múltiples referencias. Citamos aquí algunas de las más destacables: Paris, P.: «Fouilles de BeloII», Paris, 1926 pp. 165-166, 1.30 Palol, P.: «Arqueologia cristiana de la España romanaw, Madrid/ Valladolid, 1967, pp. 365 366, lám. 114. Bourgeois, A.: «Plats à reliefs d'applique de Belo» Melanges de la Casa de Velazquez, 5, 1969, p. 32 y ss. Salomonson, J. W.: «Spătrömische rote Tonware mit Reliefverzierung aus nordafrikảnischen Werkståttenn, BABesch. XLIV, 1969, Caballero Zoreda, L.: "Cerámica sigillata clara decorada de los tipos A, A/C y C" Trabajos de Prehistoria, 28, Madrid, 1971, pp. 227-262. Schlunk, H. Y Hauschild, T.: «Hispania Antiqua », Mainz am Rhein, 1978, lam. 34.
}

parece lógico pensar que a su lado se habria dispuesto la figura de Isaac, con el presunto haz de leña ${ }^{10}$, tal como aparece en el plato gaditano.

El segundo tema que encontramos representado (fragm.3) es la escena misma del sacrificio (Génesis 22,10-12). Existen varios paralelos en cerámica de esta escena, tan difundida dentro de la iconografia paleocristiana ". Marec publicó un plato aparecido en Hipona ${ }^{12}$, — de la misma forma que el de Huerta aunque de un diámetro mayor-, con el mismo tema decorativo. Probablemente ambos motivos provienen del mismo molde, al igual que ocurre con la representación en los platos de Roma (Salomonson, 1969, fig. 24) ${ }^{13}$, de Djemila (Salomonson, 1969. fig. 25) y de Bonn (Salomonson, 1969, fig. 26) y en el presentado por Salomonson como procedente de una colección particular, sin origen concreto ${ }^{14}$. En este último se puede observar la escena completa, con el carnero y el árbol del cual surge «la voz de Dios» representada como una mano; una de las formas más usuales dentro de la iconografia paleocristiana.

La escena representada en los fragmentos 4 y 5 es sin duda la más controvertida en lo que respecta a su interpretación. La estructura arquitectónica, que encontramos completa en Egipto (Salomonson 1969 , fig. 75; Atlante LXXXVI,12) y en Salomonson 1979 fig. 42, es interpretada por éste último como un horno. Ello, unido al hecho de que en ocasiones, como en el caso de éste último ejemplo citado, el fragmento de la col. Biscari, aparezcan tres figuras escapando de su interior, le ha llevado a considerar la escena como la representación del episodio biblico de los tres jóvenes hebreos(Daniel 3,8-97).

El problema de esta interpretación reside en que esta iconografia se aleja de los arquetipos que encontramos plasmados en otros soportes no cerámi-

\footnotetext{
${ }^{10}$ El problema interpretativo de esta escena existe, aunque generalizadamente se asocia con la preparación del sacrificio de Isaac. La representación del haz de leña es, en este sentido, uno de los puntos más conflictivos. La presencia de esta escena junto con la del sacrificio en si, tal como vemos en el plato de Roma representado en Salomonson, op. cit. 1969, fig. 24, podría reforzar esta interpretación.

"Ver, por ejemplo, Grabar, A.: "Las vias de la creación en la iconografia cristiana». A. f., Madrid, 1985 y Salomonson, W. J.: "Voluptatem spectandi non perdat sed mutet.Observations sur l'iconographie du martyre en Afrique romaine». Amsterdam/ Oxford/Nueva York, 1979.

12 Marec, E.: «Deux interpretations du sacrifice d'Abraham» LIBYCA, VII, 1959, pp. 147 y ss.

13 En este fragmento encontramos, al igual que ocurre en Huerta, el tema del sacrificio de Isaac en dos escenas diferentes: la representación de la escena preparatoria y la del momento álgido de la inmolación.

i4 Salomonson, op. cit., 1979, fig. 44.
} 
cos (sarcófagos, pinturas murales,,.. ) donde los tres hebreos, vestidos, con gorro frigio, se sitúan en actitud orante sobre la parrilla del horno o bien, rodeados por el fuego. El tema, muy frecuente en el arte paleocristiano, parece pues, adquirir una iconografia propia, particular en los talleres de cerámica africanos.

La interpretación de Salomonson nos parece en cualquier caso válida. No obstante, creemos que es posible considerar que existen dentro de la concepción iconográfica,algunas variantes: A nuestro juicio, hay al menos dos versiones. La primera estaría representada por los relieves del ejemplar de la col. Biscari (Salomonson 1979, fig.42) donde vemos que las figuras saliendo del horno son tres y que podemos identificar como los hebreos indemnes, dando gracias a Dios, despues del milagro. Por otro lado estaria la figuración que encontramos sobre el recipiente de Egipto ${ }^{15}$, el de Salomonson 1979 (fig. 44) y el nuestro de Huerta, donde los personajes representados serian un ángel y una figura desnuda que seguramente haya que identificar con uno de los hebreos en representación del terceto.

Las diferencias entre ambas versiones se centran primeramente en la existencia de tres figuras - los tres hebreos- o una sola acompañada de un ángel. En segundo lugar en si aparecen desnudas o vestidas $\mathrm{y}$, por último, en la presencia de un objeto más o menos circular a la altura de la cabeza del ángel y que nosotros interpretamos como la representación de la "brisa divina» que aleja el fuego de los jóvenes condenados por Nabucodonosor.

Probablemente el tema evolucionó, sin que nos atrevamos a teorizar sobre cual de las dos versiones antecedió a la otra. A nuestro entender pues, la escena representada en los fragmentos 4 y 5 hace referencia estricta a un momento concreto del episodio bíblico, tal como se narra en Daniel 3,49-50: «Pero el ángel del Señor bajó al horno con Azarías y sus compañeros, echó fuera del horno las llamas de fuego, e hizo que en medio del horno soplara como un viento de brisa, de tal modo que el fuego

\footnotetext{
is Salomonson, op. cit., 1969, fig. 75 y Atlante LXXXVI,12.
}

no los tocó en absoluto, ni les causó daño ni molestia alguna.»

En un primer momento consideramos la posibilidad de que las ocho o diez escenas que debieron decorar la totalidad del borde del plato de Huerta, tuvieran un nexo temático concreto; es decir, que hubieran formado un ciclo decorativo, por supuesto de carácter cristiano.

Tanto el sacrificio de Isaac, como el episodio de los tres hebreos en el horno, son motivos comunes, dentro de los denominados "ciclos de salvación" cuya representación más característica es la que aparece en los sarcófagos paleocristianos. Daniel y los leones, Jonás tragado por la ballena, Susana y los viejos, la resurrección de Lázaro o la curación de la hemorroisa, forman también parte de estos «ciclos de salvación» junto a los temas citados.

La intención de estos ciclos iconográficos era de plegaria fúnebre $\mathrm{y}$, al parecer, tenían su paralelismo oral y escrito en ciertas oraciones ${ }^{16}$.

Quizás fuera ésta la idea originaria que motivó la elección de los temas de decoración en este tipo de cerámica fina, pero lo cierto es que se conocen ejemplos en que la disposición anárquica de los motivos hablan de que, en ocasiones, el afán meramente ornamental se antepone a las consideraciones narrativas. El ya citado fragmento presentado por Salomonson ${ }^{17}$ muestra, en este sentido, una abigarrada y azarosa disposición de motivos, como si el único interés del artesano hubiera sido rellenar el interior del recipiente con la mayor cantidad de moldes posible. Así, aunque es cierto que la temática parece ser en todos estos casos religiosa no es posible hablar de la existencia de un ciclo narrativo concreto.

Que el resto de escenas dispuestas en el plato de Huerta fueran de tema bíblico es casi seguro. Pero pensar que formaran entre todas un ciclo narrativo, aún siendo lógico, es entrar en el terreno de la mera suposición.

16 Por ejemplo: «Padre, libra su alma, como libraste a Noe del diluvio, a Issac de las manos de Abraham, a Daniel de la fosa de los leones, a los tres hebreos del horno..." en Antioquia, según Marec op. cit., 1959, p. 147 nota 1.

17 Salomonson, op, cit., 1979, fig. 44. 
(c) Consejo Superior de Investigaciones Científicas.

http://aespa.revistas.csic.es/index.php/aespa 